\title{
IS AN INTERVAL THE RIGHT RESULT OF ARITHMETIC OPERATIONS ON INTERVALS?
}

\author{
ANDRZEJ PIEGAT ${ }^{a, *}$, MAREK LANDOWSKI $^{b}$ \\ ${ }^{a}$ Faculty of Computer Science \\ West Pomeranian University of Technology, Żołnierska 49, 71-210 Szczecin, Poland \\ e-mail: apiegat@wi.zut.edu.pl \\ ${ }^{b}$ Department of Mathematical Methods \\ Maritime University of Szczecin, Wały Chrobrego 1-2, 70-500 Szczecin, Poland \\ e-mail: m.landowski@am.szczecin.pl
}

\begin{abstract}
For many scientists interval arithmetic (IA, I arithmetic) seems to be easy and simple. However, this is not true. Interval arithmetic is complicated. This is confirmed by the fact that, for years, new, alternative versions of this arithmetic have been created and published. These new versions tried to remove shortcomings and weaknesses of previously proposed options of the arithmetic, which decreased the prestige not only of interval arithmetic itself, but also of fuzzy arithmetic, which, to a great extent, is based on it. In our opinion, the main reason for the observed shortcomings of the present IA is the assumption that the direct result of arithmetic operations on intervals is also an interval. However, the interval is not a direct result but only a simplified representative (indicator) of the result. This hypothesis seems surprising, but investigations prove that it is true. The paper shows what conditions should be satisfied by the result of interval arithmetic operations to call it a "result", how great its dimensionality is, how to perform arithmetic operations and solve equations. Examples illustrate the proposed method of interval computations.
\end{abstract}

Keywords: interval arithmetic, one-dimensional interval arithmetic, multi-dimensional interval arithmetic, RDM interval arithmetic.

\section{Introduction}

Interval arithmetic (IA, I arithmetic) is very important for artificial intelligence (in the thinking process, people use granular information), for granular computing (Pedrycz et al., 2008) and especially for fuzzy arithmetic (Pedrycz et al., 2008), because a fuzzy set can be interpreted as a sum of cuts of the set on different $\mu$-levels. It is impossible to solve many problems containing uncertainty without an effective IA. However, today it is not easy to choose an appropriate version of IA because its numerous versions have been proposed. Below, a few types of IA are presented:

- standard interval arithmetic (SIA, SI arithmetic) of Warmus-Sunaga-Moore (Moore and Young, 1959; Moore, 1996; Moore et al., 2009; Sunaga, 1958; Warmus, 1956),

\footnotetext{
*Corresponding author
}

- extended interval arithmetic of Kaucher (EIA, EI arithmetic) (Kaucher, 1980),

- affine interval arithmetic (AIA, AI arithmetic) of Figuiredo and Stolphi (Figuiredo and Stolfi, 2004),

- constrained interval arithmetic (CIA, CI arithmetic) of Lodwick (Lodwick, 1999),

- instantiation interval arithmetic (IIA, II arithmetic) of Dubois (Lodwick and Dubois, 2015).

The best known and most often used IA is SI arithmetic (SIA). It is an important step in the development of uncertainty analysis. It enabled solving many problems such as computing enclosures of solutions to uncertain computational problems and getting rigorous bounds on computational errors. There are also other reasons behind the great popularity of this arithmetic such as, e.g., its compatibility with human intuition, easiness 
in calculation and memorizing results. However, because SIA could not solve all uncertainty problems, new types of IA, as mentioned above, were developed to make progress in interval computing. But SIA has some faults. Hanss (2005), Chalco-Cano et al. (2014) and Neumaier (1990) write about overestimation of results in SIA and the dependence of the results on the form of the mathematical expression used in problem solving. Hayes (2003) and Dadala (2009) describe the "traps" of SI arithmetic. The authors write that the main reason of the observed "traps" is calculating with only borders of intervals. Moore et al. (2009) report that the arithmetic does not possess such important properties as the distributivity law and the property of multiplicative cancellation. The lack of inverse element of addition and multiplication is also very important. If $\underline{x}$ denotes the left endpoint of an interval, $\bar{x}$ stands for its right endpoint and $\bar{x} \geq \underline{x}$ (proper interval), then the interval model is given by

$$
[x]=[\underline{x}, \bar{x}], \quad \bar{x} \geq \underline{x}, \quad \underline{x}, \bar{x} \in \mathbb{R}
$$

Similarly to $[x]$, the interval $[y]$ is defined. Let $*$ be one of the operations $\{+,-, \times, \div\}$. In terms of SI arithmetic, each operation $*$ for independent variables can be defined as follows (Lodwick and Dubois, 2015):

$$
[x] *[y]=\left[\min _{x \in[x], y \in[y]} x * y, \max _{x \in[x], y \in[y]} x * y\right] .
$$

The elementary arithmetic operations $\{+,-, \times, \div\}$ are of course a basis for more complicated problems of interval computing. Therefore, they are very important. If the elementary operations are formulated imprecisely or incorrectly, then using them for solving problems can sometimes lead to controversial results. Examples of these are frequently described in the literature (e.g., Dymova, 2011; Piegat and Landowski, 2012; 2013; Piegat and Plucinski, 2015; Piegat and Tomaszewska, 2013; Sevastjanov and Dymova, 2009). One of the important problems of interval computations is interval extension $F\left(X_{1}, X_{2}, \ldots, X_{n}\right)$ of a function $f\left(x_{1}, x_{2}, \ldots, x_{n}\right)$, where $x_{1}, x_{2}, \ldots, x_{n}$ are real-valued arguments and $X_{1}, X_{2}, \cdots, X_{n}$ are interval-valued arguments. This problem can be illustrated by an example. Consider four formal expressions of the same function $f(x)$ given by

$$
\begin{array}{ll}
f_{1}(x)=x(x+1), & f_{2}(x)=x \cdot x+x, \\
f_{3}(x)=x^{2}+x, & f_{4}(x)=(x+0.5)^{2}-0.25 .
\end{array}
$$
$[-1,1]$

The expressions have been extended for $X=[x]=$

$$
\begin{aligned}
& F_{1}(X)=[x]([x]+1)=[-2,2], \\
& F_{2}(X)=[x] \cdot[x]+[x]=[-2,2], \\
& F_{3}(X)=[x]^{2}+[x]=[-1,2], \\
& F_{4}(X)=([x]+0.5)^{2}-0.25=[-0.5,2] .
\end{aligned}
$$

One can see that the accuracy of the resulting intervals obtained with SI arithmetic depends on the form of the function $f$ used in the calculations. In economies, Leontief's input-output model is of great importance (Leontief, 1949; 1966). This model is given by

$$
[A][x]=[b]
$$

where $[A]$ is an interval matrix, $[b]$ is an interval vector and $[x]$ is an interval solution vector.

In opinion of many researchers (e.g., Shary, 2002; Pilarek, 2010), the system of equations (5) has no exact solution and only approximate solutions can be determined. Even in the simplest case of $n=1$, the solution is problematical, because (5) is an interval extension of the equation $a x=b$, which has alternative forms $b / a=x, a=b / x, a x-b=0$. Particular crisp forms correspond to four interval extensions: $[a][x]=[b]$, $[b] /[a]=[x],[a][x]-[b]=0,[a]=[b] /[x]$.

Each of these interval forms delivers, in general, different results $[x]$, which means the ambiguity of the results. This phenomenon, discovered by Dymova (2011) as well as Sevastjanov and Dymova (2009), can be called the dependence of interval results on the equation form or, in short, the multiple results phenomenon (MR phenomenon). The next example of an ambiguity in interval arithmetic is the Hukuhara difference (Moore et al., 2009). According to the most commonly used SIA, the difference $[w]=[u]-[v]$ of two intervals is calculated with the formula $[\underline{w}, \bar{w}]=[\underline{u}-\bar{v}, \bar{u}-\underline{v}]$. However, for the calculation of this difference another formula, called the Hukuhara difference, is also used, i.e.,

$$
[u]=[v]+[w]^{H}:[\underline{u}, \bar{u}]=\left[\underline{v}+\underline{w}^{H}, \bar{v}+\bar{w}^{H}\right] .
$$

Thus, in the present interval arithmetic, two different formulas for the interval difference are accepted, which yield different results in general. This situation is of course very strange and unacceptable. The next disputable point of interval arithmetic is using improper intervals introduced by Kaucher (1980) in the form

$$
[a]=[\underline{a}, \bar{a}], \quad \bar{a} \leq \underline{a}, \quad \underline{a}, \bar{a} \in \mathbb{R} .
$$

Improper intervals are not accepted by Lodwick and Dubois (2015), either. Weaknesses of the present IA results in a situation when even the solution of the simplest equations as $A+X=B$ leads to difficulties and is disputed (see, e.g., Mazarhuiya et al., 2011; Kovalerchuk and Kreinovich, 2016; Piegat and Landowski, 2012) and there are different proposals of how it should be solved. What is the reason for such a situation? Earlier, five versions of interval arithmetic were mentioned: SIA, EIA, AIA, CIA, SLIA. It appears that particularly IIA and CIA seem noteworthy. They were presented in the comprehensive paper of Lodwick and Dubois (2015). For 
solving interval linear equations the authors especially recommend application of II arithmetic and CI arithmetic. Further on, a short description of these taken from the work of Lodwick and Dubois (2015) is given.

Let $* \in\{+,-, \times, \div\}$. II arithmetic is $[x] *[y]$, where

$$
[x] *[y]=\left[\min _{x \in[x], y \in[y]} x * y, \max _{x \in[x], y \in[y]} x * y\right] .
$$

The expression (8) is precisely how Moore (1996) defined interval arithmetic. Note that according to (8) the result of an arithmetic operation on intervals is also interval (1D mathematical object). IIA does not impose independence. In IIA we have

$$
[x]-[x]=[x-x]=[0,0]=0 .
$$

IIA is a different arithmetic from SIA since in the latter $[a, b]-[a, b]=[a-b, b-a]$, which equals $[x]-[y]$ if $[x]=[y]$, assuming unrelated variables $x, y$. Thus, IIA distinguishes between subtractions $[x]-[y]$ and $[x]-[y]$ even when the intervals are the same. Similarly, it can easily be seen that

$$
[x] \div[x]=[x \div[x]=[1,1]=1,
$$

if $0 \notin[x]$, which is distinct from the usual IA. IIA uses an instantiation of values inside the interval. Computing with these single instantiated values makes solving equations possible since instantiation is a real value and possesses additive and multiplicative inverses. In physical systems, the distinction between repeated intervals and independent ones, possibly of equal values, occurs when we have the same interval values coming from two different measurements or parts such as two different resistors which are outputting the same interval values in a circuit. In II arithmetic, the following epistemic view on an interval is assumed: it is an unknown value $x$ restricted by the interval $\underline{x}, \bar{x}$ denoted by $[x]$. So, $x$ is an arbitrary real number in the interval $[x]$ and we call it instantiated. If we have a different variable $y$ known to lie in the interval $[y]$, we may have that $[x]=[y]$ even if these two identical ranges refer to distinct quantities. This representation is adapted to epistemic intervals.

The description of constraint interval arithmetic (CIA) (cf. Lodwick and Dubois, 2015) is as follows: An interval may be encoded by means of a continuous and monotonic function $f\left(\lambda_{x}\right):[0,1] \rightarrow[\underline{x}, \bar{x}]$ such that

$$
f(0)=\underline{x}, \quad f(1)=\bar{x}, \quad f \text { nondecreasing },
$$

which we call a general constraint interval. We restrict ourselves for this presentation to $f\left(\lambda_{x}\right)$ being linear and increasing, that is,

$$
\begin{aligned}
f\left(\lambda_{x}\right)=\underline{x}+w_{x} \lambda_{x}, \quad w_{x}=\bar{x}-\underline{x} & \geq 0, \\
0 & \leq \lambda_{x} \leq 1
\end{aligned}
$$

called a constraint interval. It was the original approach used in the so-called constraint interval arithmetic (CIA) of Lodwick (1999). The set of constraint intervals belongs to a mathematical space that is richer in properties than the algebraic space of intervals used in SI arithmetic associated with the traditional. CI arithmetic realizes operations $\{+,-, \times, \div\}$ denoted briefly by

$$
\begin{aligned}
{[x] *[y] } & {\left[\min _{0 \leq \lambda_{x}, \lambda_{y} \leq 1}\left\{\left(\underline{x}+w_{x} \lambda_{x}\right) *\left(\underline{y}+w_{y} \lambda_{y}\right)\right\},\right.} \\
& \left.\max _{0 \leq \lambda_{x}, \lambda_{y} \leq 1}\left\{\left(\underline{x}+w_{x} \lambda_{x}\right) *\left(\underline{y}+w_{y} \lambda_{y}\right)\right\}\right],
\end{aligned}
$$

where $* \in\{+,-, \times, \div\}$. It appears that a constraint interval merely transforms $x \in[a, b]$ into $x=a+(b-a) \lambda$, for some $\lambda \in[0,1]$. If it is true, one thinks of transforming a particular single value $x$. However, a constraint interval as defined by (11) is the function $f\left(\lambda_{x}\right)$ whose domain is $0 \leq \lambda_{x} \leq 1$ that explicitly keeps track of dependences and lack of dependences all in one representation and operates on the level of expressions. In CIA, we have

$$
\begin{gathered}
{[x]-[x]=\left[f\left(\lambda_{x}\right)-f\left(\lambda_{x}\right)\right]=[0,0]=0,} \\
{[x] \div[x]=\left[f\left(\lambda_{x}\right) \div f\left(\lambda_{x}\right)\right]=[1,1] \quad \text { if } 0 \notin[x] .}
\end{gathered}
$$

IIA and CIA also have the following property in contrast to the standard:

$$
[x]([y]+[z])=[x][y]+[x][z] .
$$

Note that, according to CI arithmetic, the result of an arithmetic operation on intervals is also an interval.

\section{Important demands on interval arithmetic}

A very important demand on the arithmetic is the possibility of transformations of formulas (mathematical models, $M$ models) to enable the calculation of any variable (parameter) occurring in the $\mathrm{M}$ model which we want to determine. For example, if a model of a dependence existing in a system has the form $a+x=b$ and we know $a \in[\underline{a}, \bar{a}]$ and $b \in[\underline{b}, \bar{b}]$, then we want to determine the unknown $x$ by a formula transformation. The result $x$ has to be verifiable. How can we proceed with the verification in the simplest way? By substituting the result into the original equation (mathematical model). Such a method is very well known under the name of an algebraic solution (Lyashko, 2005; Popova, 1998; Shary, 1996). For interval computations, Popova (1998) gives the following definition: the interval algebraic solution of a linear interval equation is an interval (interval vector) such that substituting it into the equation(s) and performing all interval operations results in valid equality(ies). Let us 
notice that according to Popova (1998) solution of a linear interval equation is an interval (interval vector), i.e., the same mathematical object type.

A similar definition is given by Lyashko (2005): An interval vector is called an algebraic solution of the interval equation if after substituting this vector into the given equation and executing all interval operations according to the rules of interval arithmetic we get an equality. However, we would like to extend this definition taking into account the multiple results phenomenon (MR phenomenon). The proposal of an extended definition is as follows: the algebraic solution is a solution such that substituting it into the pertinent system of equations and performing all interval operations according to the rules of interval arithmetic, we get an equality of both the sides of all the equations independently of the mathematical form of the system of equations used in calculations. Thus, an algebraic solution (result) should satisfy the (result) universality condition for the solution.

Eliminating the ambiguity of results from interval arithmetic is a basic matter, which conditions its scientific credibility. As Dymova (2011) as well as Sevastjanov and Dymova (2009) have shown, the standard IA is incredible in this respect, because depending on the given form of a system model it delivers different results. However, it does not mean that SIA is of no value because this arithmetic allows solving many problems and has many practical applications (Moore et al., 2009). We would only like to say that the application of this arithmetic is limited.

Now, investigate the credibility of the new versions of IA, i.e., of instantiated IA and of constrained IA. In our opinion, these arithmetic versions undoubtedly mean a progress in interval computations. This was shown by Lodwick and Dubois (2015). However, it seems that these arithmetic versions are not ideal and can be improved because they do not deliver universal algebraic solutions. Let us consider the simple, basic operation of interval addition $[x]+[y]$ and its result suggested both by SIA, IIA and CIA. This operation can be interpreted as the dependence existing in a real system in which the output $z$ is the sum of two inputs $x$ and $y$ but their values are known only approximately in the form of constraints given by intervals $[x]$ and $[y]$. An example of such a system can be a tank with two water inflows or a firm obtaining incomes from two sources. The operation of addition of two proper intervals $[x]$ and $[y]$ gives, according to SIA, IIA, CIA, an identical result, expressed by

$$
[\underline{x}, \bar{x}]+[\underline{y}, \bar{y}]=[\underline{x}+\underline{y}, \bar{x}+\bar{y}]=[\underline{z}, \bar{z}] .
$$

Formulas confirming that statement can be found, e.g., in the work of Lodwick and Dubois (2015, p. 9) and Lodwick (1999). Let us notice that the formula (16) suggests that the result of addition of two intervals (interval numbers (Lodwick, 1999)) is also an interval, that is, the same mathematical object. Such a result is consistent with the axiom of closure that can be formulated as follows: a set of intervals has a closure under an operation (e.g., of addition) if this operation on members of the set always produces a member of the same set, i.e., an interval. Such an interval set is said to satisfy the closure property (Bader and Nipkow, 1998; Birkhoff, 1967). From an intuitive point of view, the closure property seems obvious and perhaps this axiom has motivated the creators of existing versions of interval arithmetic to make the assumption that the result of an operation on interval is also an interval. Now, let us check whether a result of intervals' addition (16) is the algebraic result of all possible interval extensions of the addition operation $x+y=z$. If a real system is ruled by dependence $x+y=z$ then it is also ruled by transformed dependences given under

$$
\begin{aligned}
x+y=z, \quad x= & z-y, \\
& y=z-x, \quad x+y-z=0 .
\end{aligned}
$$

Interval extensions of crisp forms of addition are given by

$$
\begin{gathered}
{[x]+[y]=[z],} \\
{[x]=[z]-[y],} \\
{[y]=[z]-[x],} \\
{[x]+[y]-[z]=0 .}
\end{gathered}
$$

The result of interval addition (18) according to SIA, IIA and CIA is the interval $[z]$ determined by (16). Now, let us check the extension (19) given by (22):

$$
\begin{aligned}
{[x] } & =[z]-[y]=[z]+(-[y]), \\
{[\underline{x}, \bar{x}] } & =[\underline{z}, \bar{z}]+(-[\underline{y}, \bar{y}]) \\
& =[\underline{z}, \bar{z}]+[-\bar{y},-\underline{y}] \\
& =[\underline{z}-\bar{y}, \bar{z}-\underline{y}], \\
\underline{x} & =\underline{z}-\bar{y}: \underline{z}=\underline{x}+\bar{y} \\
\bar{x} & =\bar{z}-\underline{y}: \bar{z}=\bar{x}+\underline{y} \\
{[\underline{z}, \bar{z}] } & =[\underline{x}+\bar{y}, \bar{x}+\underline{y}] .
\end{aligned}
$$

The result (22) is different from that suggested by the definitions of the interval addition (16) according to SIA, IIA and CIA. Now, let us consider the extension (20) of the interval addition given by

$$
\begin{aligned}
{[y] } & =[z]-[x]=[z]+(-[x]), \\
{[\underline{y}, \bar{y}] } & =[\underline{z}, \bar{z}]+(-[\underline{x}, \bar{x}]) \\
& =[\underline{z}, \bar{z}]+[-\bar{x},-\underline{x}], \\
& =[\underline{z}-\bar{x}, \bar{z}-\underline{x}] \\
\underline{y} & =\underline{z}-\bar{x}: \underline{z}=\underline{y}+\bar{x}, \\
\bar{y} & =\bar{z}-\underline{x}: \bar{z}=\bar{y}+\underline{x}, \\
{[\underline{z}, \bar{z}] } & =[\bar{x}+\underline{y}, \underline{x}+\bar{y}] .
\end{aligned}
$$


The result (23) is also different from (16) suggested by the definitions of interval addition according to the SIA, IIA and CIA. Now, lets us consider the extension 21 of interval addition given by

$$
\begin{gathered}
{[x]+[y]-[z]=0,} \\
{[\underline{x}, \bar{x}]+[\underline{y}, \bar{y}]+(-[\underline{z}, \bar{z}])=0,} \\
{[\underline{x}+\underline{y}, \bar{x}+\bar{y}]+[-\bar{z},-\underline{z}]=0,} \\
{[\underline{x}+\underline{y}-\bar{z}, \bar{x}+\bar{y}-\underline{z}]=[0,0]=0,} \\
\underline{x}+\underline{y}-\bar{z}=0: \bar{z}=\underline{x}+\underline{y}, \\
\bar{x}+\bar{y}-\underline{z}=0: \underline{z}=\bar{x}+\bar{y}, \\
{[z]=[\bar{x}+\bar{y}, \underline{x}+\underline{y}] .}
\end{gathered}
$$

This result is also different from (16). Summarizing there exist four possible results of addition of intervals dependent on four possible mathematical forms of the addition operation, namely,

$$
\begin{aligned}
& {[\underline{x}, \bar{x}]+[\underline{y}, \bar{y}]=[\underline{z}, \bar{z}]=[\underline{x}+\underline{y}, \bar{x}+\bar{y}],} \\
& {[\underline{x}, \bar{x}]+[\underline{y}, \bar{y}]=[\underline{z}, \bar{z}]=[\underline{x}+\bar{y}+\underline{y}],} \\
& {[\underline{x}, \bar{x}]+[\underline{y}, \bar{y}]=[\underline{z}, \bar{z}]=[\bar{x}+\underline{y}, \underline{x}+\overline{\bar{y}}],} \\
& {[\underline{x}, \bar{x}]+[\underline{y}, \bar{y}]=[\underline{z}, \bar{z}]=[\bar{x}+\overline{\bar{y}}, \underline{x}+\underline{y}] .}
\end{aligned}
$$

This situation cannot be accepted. None of the four possible results 25) is a universal result of all four possible forms of the interval addition (18)-(22). A similar situation exists in the case of other arithmetic operations: subtraction, multiplication and division.

The conclusion is that the analysed formulations of interval arithmetic contain a certain basic error being the reason of the observed controversial results. In our opinion, the error stems from the generally incorrect approach to interval arithmetic. An approach that we think is correct will be presented in Section 3

\section{Proposed approach to interval arithmetic}

In our opinion, reasons for controversial results observed in the present versions of interval arithmetic are as follows:

- Calculations in the present versions of IA are realized only with the use of intervals, while they should be realized with the use of $\mathrm{M}$ models of precise values of uncertain variables (parameters). Note: One should distinguish between the precise (true) value of a variable and the $\mathrm{M}$ model of this value. If we have the $\mathrm{M}$ model, this does not mean that we know the precise value of the variable itself. The calculation result is denoted by $z^{\mathrm{pr}}$. It allows generating all possible point-results $z^{\text {poss }}$ creating the set $Z^{\text {poss }}$ that gives the information about uncertainty in the precise result $z^{\text {pr }}$.
- In present versions of IA the notion of the solution (result) of a calculation is sometimes used in relation to partial sets (subsets) of the complete solution (result) set (CSS or CS set) and in relation to simplified representatives of this set. However, these notions should be distinguished. Representatives of the CS set give simple information about it, mostly about its uncertainty, but they should not be used in further steps of computation algorithms. In such algorithms, computations should be realized with models of precise results to get the final solution. Then representatives of this solution that we are interested in can be determined.

- Present versions of IA do not satisfy an important requirement that can be called the principle of solution (result) universality. This principle can be formulated as follows: the mathematical solution of a problem (an equation, a system of equations) has to be of universal algebraic character, which means that after substituting it into the $M$ model the equality of the right- and left-hand sides of the model is achieved. This also means that regardless of which mathematical form of the model the solution (result) has been determined it should have the same mathematical form.

In the case of interval calculations concerning a problem with variables $x, y$ and parameter $a$, the procedure should be as follows:

Step 1. Determine a mathematical model $F(x, y, a)=0$ of the system in a traditional form, i.e., appropriate for calculations with precise, crisp values of all variables and parameters.

Step 2. By model transformations derive a formula for the variable (parameter) you are interested in, e.g., $y=$ $f_{y}(x, a)$.

Step 3. In the model $y=f_{y}(x, a)$ introduce RDM models of precise values of uncertain variables and parameters $x\left(\alpha_{x}\right), a\left(\alpha_{a}\right)$. The obtained model $y^{\mathrm{pr}}=$ $f_{y}\left(x\left(\alpha_{x}\right), a\left(\alpha_{a}\right)\right)$ is called the model of precise variable value (PVV model) and $\alpha_{x}, \alpha_{a}$ are RDM variables. The PVV model allows generating all possible point solutions to the problem.

Step 4. On the basis of the PVV model determine the model $y^{\text {poss }}=f_{y}\left(x\left(\alpha_{x}\right), a\left(\alpha_{a}\right)\right)$ being the $\mathrm{M}$ model of the algebraic solution set and determine its subsets or representatives that are of your interest.

\section{Mathematical model of the precise variable value represented by an interval}

If a precise value of variable $x$ is unknown but we have approximate knowledge that it is contained in an interval 
$[x]=[\underline{x}, \bar{x}]$, then the following model (PVV model) represents the precise, true value of the variable that occurred in the real system:

$$
\begin{aligned}
& X^{\mathrm{pr}}: x^{\mathrm{pr}}=\underline{x}+\alpha_{x}(\bar{x}-\underline{x}), \\
& \alpha_{x} \in[0,1], \quad \operatorname{card} X=1,
\end{aligned}
$$

$X^{\mathrm{pr}}$ denotes the set of true $x$-values. Since only one value could occur in the system, cardinality of this set is equal to 1 . Hence, this set is of epistemic character (Lodwick and Dubois, 2015). The RDM variable $\alpha_{x}$ informs about the relative position of a chosen value of variable $x$ in the interval $[x]$. Though the formula (26) is called the PVV model, this does not mean that the precise $x$-value $x^{\mathrm{pr}}$ is known. The formula (26) informs that only one true value exists between $\underline{x}$ and $\bar{x}$. From the PVV model of $x^{\mathrm{pr}}$ (26), one should distinguish the model of $X^{\text {poss }}$ that allows generating all possible values of the set $X^{\text {poss }}$ :

$$
\begin{aligned}
& X^{\text {poss }}: x^{\text {poss }}=\underline{x}+\alpha_{x}^{\text {poss }}(\bar{x}-\underline{x}), \\
& \alpha_{x}^{\text {poss }} \in[0,1], \quad \text { card } X^{\text {poss }}=c(\text { continuum }) .
\end{aligned}
$$

The difference between the sets $X^{\text {poss }}$ and $X^{\text {pr }}$ consist in the meaning and cardinality. The set of precise, true values of $X^{\mathrm{pr}}$ contains only one value and is of epistemic character and the set $X^{\text {poss }}$ contains a continuum of possible values $x^{\text {poss }}$ and is of ontic character (Lodwick and Dubois, 2015). The model (26) of the precise value $x^{\mathrm{pr}}$ was introduced in the framework of multidimensional RDM arithmetic by Piegat and Landowski (2012) without any knowledge of CI arithmetic. A similar model of an instantiated $x$-value was earlier introduced by Lodwick in the framework of CI arithmetic. However, the general idea of both the types of interval arithmetic is different, first of all in understanding of arithmetic operations, which will be explained further on. The RDM is an abridgement of relative distance measure. In the traditional coordinate system each value of a variable $x$ is positioned. However, in the traditional interval notation $[\underline{x}, \bar{x}]$, we cannot position particular values of variable $x$. The RDM variable $\alpha_{x} \in[0,1]$ allows positioning each $x$-value because it introduces the RDM coordinate system with a unit of the variable, similarly as in the traditional Cartesian coordinate system. RDM variables have the meaning of normalized coordinates determining the distance of a single $x$-value from the origin of the local coordinate system. The meaning of RDM coordinates $\alpha_{x 1}, \alpha_{x 2} \in[0,1]$ is illustrated in Fig.1]

\section{RDM interval arithmetic}

The main difference between RDM-IA and other arithmetic types consists in the fact that RDM-IA operations are realized not on intervals (sets) but on models of precise variable values. Hence, the achieved

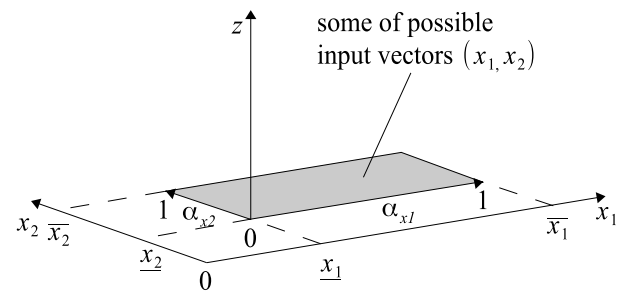

Fig. 1. RDM variables $\alpha_{x 1}, \alpha_{x 2} \in[0,1]$ as coordinates of a local, normalized coordinate system formed by intervals.

result is also a model of the precise variable value and not an interval. The result is multidimensional and not one-dimensional. Let us denote by $x^{\mathrm{pr}}$ and $y^{\mathrm{pr}}$ precise, true values of variables about which we possess only approximate knowledge $x^{\mathrm{pr}} \in[\underline{x}, \bar{x}]$ and $y^{\mathrm{pr}} \in[\underline{y}, \bar{y}]$, and by $*$ one of the arithmetic operations in $\{+,-, \times, \div\}$. Models of precise values of variables $x$ and $y$ are given by

$$
\begin{aligned}
& x^{\mathrm{pr}}=\underline{x}+\alpha_{x}(\bar{x}-\underline{x}), \\
& \alpha_{x} \in[0,1], \quad \operatorname{card} X^{\mathrm{pr}}=1, \\
& y^{\mathrm{pr}}=\underline{y}+\alpha_{y}(\bar{y}-\underline{y}), \\
& \alpha_{y} \in[0,1], \quad \operatorname{card} Y^{\mathrm{pr}}=1 .
\end{aligned}
$$

A model of the precise result value $z^{\mathrm{pr}}$ of any arithmetic operation $*$ is formulated by

$$
\begin{aligned}
& z^{\mathrm{pr}}\left(\alpha_{x}, \alpha_{y}\right)=x^{\mathrm{pr}}\left(\alpha_{x}\right) * y^{\mathrm{pr}}\left(\alpha_{y}\right), \\
& \alpha_{x}, \alpha_{y} \in[0,1], \quad \operatorname{card} X^{\mathrm{pr}}=1, \quad \operatorname{card} Y^{\mathrm{pr}}=1
\end{aligned}
$$

Let us notice that the result $z^{\mathrm{pr}}\left(\alpha_{x}, \alpha_{y}\right)$ is multidimensional and not one-dimensional. It is the $\mathrm{M}$ model of the precise value. Now, let us test on an example of interval addition whether the result (30) has properties of the algebraic and universal result with respect to all four possible forms of addition (18)-(21). The result of addition $z^{\mathrm{pr}}$ on the basis of (30) has the form

$$
\begin{aligned}
& z^{\mathrm{pr}}=\left[\underline{x}+\alpha_{x}(\bar{x}-\underline{x})\right]+\left[\underline{y}+\alpha_{y}(\bar{y}-\underline{y})\right] \\
& \alpha_{x}, \alpha_{y} \in[0,1], \quad \operatorname{card} X^{\mathrm{pr}}=1, \quad \operatorname{card} Y^{\mathrm{pr}}=1 .
\end{aligned}
$$

The basic addition formula has the form $x+y=z$, substituting (28) into (18) and (29) and (31) into (18), we obtain

$$
\begin{aligned}
& \left\{\left[\underline{x}+\alpha_{x}(\bar{x}-\underline{x})\right]+\left[\underline{y}+\alpha_{y}(\bar{y}-\underline{y})\right]\right\} \\
& \quad=\left[\underline{x}+\alpha_{x}(\bar{x}-\underline{x})\right]+\left[\underline{y}+\alpha_{y}(\bar{y}-\underline{y})\right] \\
& \alpha_{x}, \alpha_{y} \in[0,1], \quad \operatorname{card} X^{\mathrm{pr}}=1, \quad \operatorname{card} Y^{\mathrm{pr}}=1 .
\end{aligned}
$$

The second possible form of addition $x+y=z$ is 
the form (19), $x=z-y$. Substituting (31) in it, we get

$$
\begin{aligned}
& {[\underline{x}+}\left.\alpha_{x}(\bar{x}-\underline{x})\right] \\
&=\left\{\left[\underline{x}+\alpha_{x}(\bar{x}-\underline{x})\right]+\left[\underline{y}+\alpha_{y}(\bar{y}-\underline{y})\right]\right\} \\
&-\left[\underline{y}+\alpha_{y}(\bar{y}-\underline{y})\right] \\
& \alpha_{x}, \alpha_{y} \in[0,1], \quad \operatorname{card} X^{\mathrm{pr}}=1, \quad \operatorname{card} Y^{\mathrm{pr}}=1 .
\end{aligned}
$$

After the reduction of the term $y^{\mathrm{pr}}$ on its right-hand side, we obtain

$$
\begin{aligned}
& {\left[\underline{x}+\alpha_{x}(\bar{x}-\underline{x})\right]=\left[\underline{x}+\alpha_{x}(\bar{x}-\underline{x})\right],} \\
& \alpha_{x} \in[0,1], \quad \operatorname{card} X^{\mathrm{pr}}=1 .
\end{aligned}
$$

The third possible form of addition $x+y=z$ is (20), i.e., $y=z-x$. Substituting (31) in it, we obtain

$$
\begin{aligned}
& {\left[\underline{y}+\alpha_{y}(\bar{y}-\underline{y})\right]} \\
& \quad=\left\{\left[\underline{x}+\alpha_{x}(\bar{x}-\underline{x})\right]+\left[\underline{y}+\alpha_{y}(\bar{y}-\underline{y})\right]\right\} \\
& \quad-\left[\underline{x}+\alpha_{x}(\bar{x}-\underline{x})\right] \\
& \alpha_{x}, \alpha_{y} \in[0,1], \quad \operatorname{card} X^{\mathrm{pr}}=1, \quad \operatorname{card} Y^{\mathrm{pr}}=1 .
\end{aligned}
$$

After the reduction $x^{\mathrm{pr}}\left(\alpha_{x}\right)$ on the right-hand side, we get

$$
\begin{aligned}
{\left[\underline{y}+\alpha_{y}(\bar{y}-\underline{y})\right]=} & {\left[\underline{y}+\alpha_{y}(\bar{y}-\underline{y})\right], } \\
& \alpha_{y} \in[0,1], \quad \operatorname{card} Y^{\mathrm{pr}}=1 .
\end{aligned}
$$

The fourth possible form of addition $x+y=z$ is (21), i.e., $x+y-z=0$. Substituting (31) in it, we have

$$
\begin{aligned}
& {\left[\underline{x}+\alpha_{x}(\bar{x}-\underline{x})\right]+\left[\underline{y}+\alpha_{y}(\bar{y}-\underline{y})\right]} \\
& \quad-\left\{\left[\underline{x}+\alpha_{x}(\bar{x}-\underline{x})\right]+\left[\underline{y}+\alpha_{y}(\bar{y}-\underline{y})\right]\right\}=0, \\
& \alpha_{x}, \alpha_{y} \in[0,1], \quad \operatorname{card} X^{\mathrm{pr}}=1, \quad \operatorname{card} Y^{\mathrm{pr}}=1 .
\end{aligned}
$$

After the reduction on the right-hand side of the equation, we achieve the indentity of both the sides, $0=0$. The above analysis has shown that the result (31) of addition of interval-valued variable values has properties of the algebraic result and satisfies the universality principle. Just as for addition, also the correctness and universality of results can easily be proved for other arithmetic operations realized according to (31). The formula (31) determines the mathematical model of the precise result of arithmetic operations. The set $Z^{\text {pr }}$ contains only one element because in a real system only one state determined by a triple $\left(x^{\mathrm{pr}}, y^{\mathrm{pr}}, z^{\mathrm{pr}}\right)$ occurs in a given, single instant. However, in practical problems we are interested in the set $Z^{\text {poss }}$ containing all point results which can occur in a system when our knowledge about inputs $x, y$ is approximate and of interval character. Determining this set is very important for the evaluation of uncertainty of the result. When the set $Z^{\text {pr }}$ of precise values that occurred in a real system is epistemic and



Fig. 2. Visualisation of the set $Z^{\text {poss }}$ of possible point results of addition $x^{\text {poss }}+y^{\text {poss }}=z^{\text {poss }}, x^{\text {poss }} \in[\underline{x}, \bar{x}]=[1,3]$, $y^{\text {poss }} \in[y, \bar{y}]=[2,5]$ of independent variable values in the 3D-space $X \times Y \times Z$.

contains only one-element $\left(\operatorname{card} Z^{\mathrm{pr}}=1\right)$, then the set of possible results $Z^{\text {poss }}$ contains an infinite number of elements (ontic set) and its cardinality $\operatorname{card}\left(Z^{\text {poss }}\right)=$ $c$ (continuum). The set $Z^{\text {poss }}$ is given by

$$
\begin{aligned}
& Z^{\text {poss }}: z^{\text {poss }}=x^{\text {poss }}\left(\alpha_{x}\right)+y^{\text {poss }}\left(\alpha_{y}\right), \\
& \alpha_{x}, \alpha_{y} \in[0,1], \quad \text { card } X^{\text {poss }}, \quad \text { card } Y^{\text {poss }}=c,
\end{aligned}
$$

where

$$
\begin{aligned}
& X^{\text {poss }}: x^{\text {poss }}=\underline{x}+\alpha_{x}(\bar{x}-\underline{x}), \\
& Y^{\text {poss }}: y^{\text {poss }}=\underline{y}+\alpha_{y}(\bar{y}-\underline{y}) .
\end{aligned}
$$

The set $Z^{\text {poss }}$ is presented in Fig. 2 .

Figure 2 concerns addition of two interval-valued variables $x$ and $y$ about which we know that $x \in$ $[\underline{x}, \bar{x}]=[1,3]$ and $y \in[y, \bar{y}]=[2,5]$. Equation (38) is a mathematical model of the set $Z^{\text {poss }}$ which enables generating all possible values of $z^{\text {poss }}$ for given possible values of $x^{\text {poss }}$ and $y^{\text {poss }}$ or, in other words, enables generating all possible states of a system that is ruled by the dependence $z=x+y$. If $x^{\text {poss }} \in[1,3]$ and $y^{\text {poss }} \in$ $[2,5]$, then in terms of RDM arithmetic $x^{\text {poss }}=1+2 \alpha_{x}$, $\alpha_{x} \in[0,1]$ and $y^{\text {poss }}=2+3 \alpha_{y}, \alpha_{y} \in[0,1]$. Assuming $\alpha_{x}=0.1$ and $\alpha_{y}=0.3$, the following system state is achieved: $x^{\text {poss }}=1.2, y^{\text {poss }}=2.6$ and $z^{\text {poss }}=3.8$. The triple $\left(x^{\text {poss }}, y^{\text {poss }}, z^{\text {poss }}\right)=(1.2,2.6,3.8)$ determines one of possible system states.

The other allowed values of $\alpha_{x}$ and $\alpha_{y}$ permit generating other possible system states, among them possible values of the result $z^{\text {poss }}$. The set $Z^{\text {poss }}$ itself is not the result of addition of uncertain interval-valued variables. The addition result is $z^{\mathrm{pr}}$ determined by (30). The set $Z^{\text {poss }}$ is only used for uncertainty evaluation of the result $z^{\mathrm{pr}}$ and should not be used in possible further calculations. Only one of the points lying on the surface of the set $Z^{\text {poss }}$ (Fig. 2) corresponds to the precise, true values $\left(x^{\mathrm{pr}}, y^{\mathrm{pr}}, z^{\mathrm{pr}}\right)$ which occurred in the real system. 


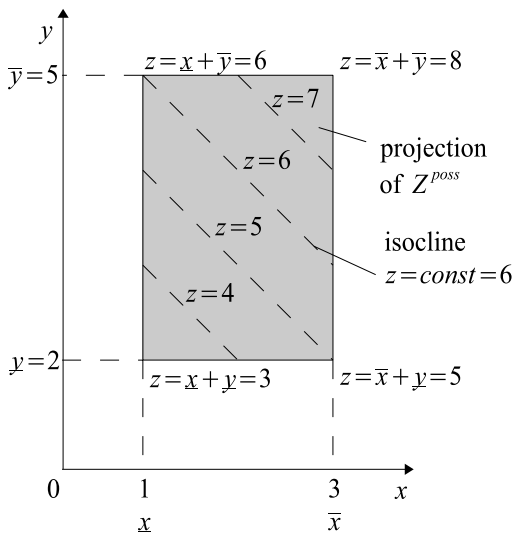

Fig. 3. Projection of the set of possible results $Z^{\text {poss }}$ from the 3D-space $X \times Y \times Z$ on the 2D-space $X \times Y$.

As Fig. 2 shows, the set of possible addition results is the information granule existing in a $3 \mathrm{D}$-space: the resulting set is not a one-dimensional interval. Simplifying the set $Z^{\text {poss }}$ to a one-dimensional interval, as happens in many I arithmetic versions, yields a considerable information loss. Observe in Fig. 2 that the subset of addition results for $z^{\text {poss }}=8$ consists of only one element (one possible system state), whereas for $z^{\text {poss }}=6$ it has the cardinality of a continuum.

\section{Set of possible results $Z^{\text {poss }}$ of arithmetic operations and simplified representatives of this set}

The set of possible results of an arithmetic operation $*$ and, in particular, the set of possible results of addition determined by (39) and shown in Fig. 2 can be difficult to interpret because they exist in a 3D-space (Fig. 3 shows its projection on a $2 \mathrm{D}$-space). Therefore, to give better and more understandable information about this set, various simplified representatives of it are used. There is nothing wrong in this approach. However, such simplified representatives are frequently called a "result." This can be observed in all present versions of interval arithmetic:

$$
\begin{aligned}
& Z^{\text {poss }}: z^{\text {poss }}=x^{\text {poss }}\left(\alpha_{x}\right)+y^{\text {poss }}\left(\alpha_{y}\right) \\
& \alpha_{x}, \alpha_{y} \in[0,1], \quad \text { card } X^{\text {poss }}, \quad \text { card } Y^{\text {poss }}=c \\
& x^{\text {poss }}=1+2 \alpha_{x}, \quad y^{\text {poss }}=2+3 \alpha_{y} \\
& z^{\text {poss }}=\left(1+2 \alpha_{x}\right)+\left(2+3 \alpha_{y}\right)
\end{aligned}
$$

The most commonly used representative is the span $s\left(Z^{\text {poss }}\right)$ of possible point-results. The span of an arithmetic operation $*$ on interval-valued variables is



Fig. 4. Non-normalized distribution of cardinality measure $M \operatorname{card}(z)$ of particular possible addition results $z=$ $x+y=$ const (representative, indicator).

expressed by

$$
\begin{aligned}
s\left(Z^{\mathrm{poss}}\right)= & {\left[\min _{\alpha_{x}, \alpha_{y} \in[0,1]} z^{\text {poss }}\left(\alpha_{x}, \alpha_{y}\right),\right.} \\
& \left.\max _{\alpha_{x}, \alpha_{y} \in[0,1]} z^{\text {poss }}\left(\alpha_{x}, \alpha_{y}\right)\right] \\
= & {\left[\min _{\alpha_{x}, \alpha_{y} \in[0,1]}\left(x^{\text {poss }}\left(\alpha_{x}\right) * y^{\text {poss }}\left(\alpha_{y}\right)\right),\right.} \\
& \left.\max _{\alpha_{x}, \alpha_{y} \in[0,1]}\left(x^{\text {poss }}\left(\alpha_{x}\right) * y^{\text {poss }}\left(\alpha_{y}\right)\right)\right] .
\end{aligned}
$$

In the case of addition, the span of the set $Z^{\text {poss }}$ (Fig. 2) is given by (41). The meaning of the span in relation to the full set $Z^{\text {poss }}$ can be easily understood from Fig. 2

$$
s\left(Z^{\text {poss }}\right)=[\underline{x}+\underline{y}, \bar{x}+\bar{y}] .
$$

The span $s\left(Z^{\text {poss }}\right)$ as secondary simplified information about the set $Z^{\text {poss }}$ (indicator, representative) is not a result of an arithmetic operation and should not be used in possible further calculations. The other simplified but better information about the set $Z^{\text {poss }}$ can be the cardinality of possible point results $z$. Figure 2 shows isoclines (lines of constant $z$-values, e.g., $z=4$, $z=5$ ). Isoclines show which values of the result $z$ of an arithmetic operation have higher or lower frequency. As the cardinality measure $M \operatorname{card}(z)$, can the length $L(z=$ const $)$ of the segments corresponding to particular subsets $z=$ const be assumed. In the case of binary arithmetic operations for cardinality determination one can use both a 3D-visualisation as in Fig. 2 and a projection of the set $Z^{\text {poss }}$ on the 2D-space $X \times Y$ (Fig. 3).

On the basis of Fig. 3, cardinality measures can easily be calculated: $M \operatorname{card}(z=8)=0, M \operatorname{card}(z=4)=$ $\sqrt{2}, M \operatorname{card}(z=5)=\sqrt{8}, M \operatorname{card}(z=6)=\sqrt{8}$, $M \operatorname{card}(z=8)=0$.

The distribution of cardinality measures of possible addition results $z=$ const is shown in Fig. 4

The distribution $M \operatorname{card}(z)$ is very valuable information about the frequency of possible results $z^{\text {poss }}$. 


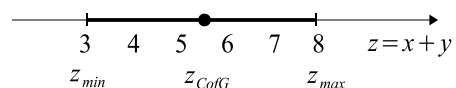

Fig. 5. Center of gravity CofG of the set $Z^{\text {poss }}$ of possible addition of interval-valued uncertain variables.

However, it represents only the set $Z^{\text {poss }}$ of possible point results and is not a direct addition result itself. In conceivable further calculations, it should not be substituted as the result. The right result is given by (39). The next representative of the set $Z^{\text {poss }}$ can be the center of gravity $z_{\text {CofG }}$ of this set, which can be calculated from

$$
Z_{\mathrm{CofG}}=\frac{\int_{\min z}^{\max z} z \cdot M \operatorname{card}(z) \mathrm{d} z}{\int_{\min z}^{\max z} M \operatorname{card}(z) \mathrm{d} z} .
$$

In the case of the addition example considered, $z_{\text {CofG }}=5.5$. The result representative, the center of gravity, can be shown together with a span $s\left(Z^{\text {poss }}\right)$ of the set of all possible point-results as in Fig. 5

Interval RDM arithmetic allows correct realization of arithmetic operations for all fully independent variables, partly dependent and fully dependent variables. Let us consider partial dependence of variables $x$ and $y$. An example of such dependence can be the relation $y \geq$ $x$. In terms of RDM arithmetic, it can be expressed as $\underline{y}+\alpha_{y}(\bar{y}-\underline{y}) \geq \underline{x}+\alpha_{x}(\bar{y}-\underline{y}), \alpha_{x}, \alpha_{y} \in[0,1]$. In this case, the formula for the precise result $z^{\mathrm{pr}}$ arithmetic operations $* \in\{+,-, \times, \div\}$ is given by

$$
\begin{aligned}
& z^{\mathrm{pr}}\left(\alpha_{x}, \alpha_{y}\right)=x^{\mathrm{pr}}\left(\alpha_{x}\right) * y^{\mathrm{pr}}\left(\alpha_{y}\right), \\
& \alpha_{y} \geq\left[\underline{x}-\underline{y}+\alpha_{x}(\bar{y}-y)\right] /(\bar{y}-\underline{y}), \\
& \alpha_{x}, \alpha_{y} \in[0,1], \quad \operatorname{card} \bar{X}^{\mathrm{pr}}, \quad \operatorname{card} Y^{\mathrm{pr}}=1 .
\end{aligned}
$$

Instead, the formula presenting a mathematical model of the set $Z^{\text {poss }}$ of possible point results is given by

$$
\begin{aligned}
& Z^{\text {poss }}: x^{\text {poss }}\left(\alpha_{x}\right) * y^{\text {poss }}\left(\alpha_{y}\right), \\
& \alpha_{y} \geq\left[\underline{x}-\underline{y}+\alpha_{x}(\bar{y}-y)\right] /(\bar{y}-\underline{y}) \\
& \alpha_{x}, \alpha_{y} \in[0,1], \quad \text { card } \bar{X}^{\text {poss }}, \quad \text { card } Y^{\text {poss }}=c .
\end{aligned}
$$

In the case of addition of two uncertain interval-valued numbers $x \in[1,3]$ and $y \in[2,5]$, the addition formula takes the form

$$
\begin{aligned}
& Z^{\text {poss }}: z^{\text {poss }}=\left(1+2 \alpha_{x}\right)+\left(2+3 \alpha_{y}\right), \\
& \alpha_{y} \geq-1 / 3+2 / 3 \cdot \alpha_{x}, \quad \alpha_{x}, \alpha_{y} \in[0,1], \\
& \text { card } X^{\text {poss }}, \quad \text { card } Y^{\text {poss }}=c .
\end{aligned}
$$

If the addition is made with SIA (2), IIA (8) or $\mathrm{CI}$ arithmetic (12), then the addition result has the form of an interval representative $z^{\text {poss }}=[3,8]$. It is a correct $\operatorname{span} s\left(Z^{\text {poss }}\right)$ of the addition under the condition



Fig. 6. Set $Z^{\text {poss }}$ of point results of addition of partly dependent variables $x^{\text {poss }} \in[\underline{x}, \bar{x}]=[1,3]$ and $y^{\text {poss }} \in[\underline{y}, \bar{y}]=$ $[2,5]$ under condition $y \geq x$ in projection on the $X \times Y$ space (cf. Fig. 3).

$y \geq x$. However, the identical "addition result" is achieved when intervals $[1,3]$ and $[2,5]$ are added without the condition $y \geq x$, when variables $x$ and $y$ are independent. Thus, dependence of variables has no influence on the "addition result" in SIA, IIA and CI arithmetic. This situation is due to the fact that these types of I arithmetic "do not notice" this dependence because they "assume" an interval to be a direct result of arithmetic operations. Instead, multidimensional RDM arithmetic perceives all dependences (relations) existing between variables (see Fig. 6).

Now, let us consider the case of full dependence of variables $x=y$ (we know that uncertain variable values are equal) on the example of addition of $x \in[1,3]$ and $y \in[2,5]$. The condition $x=y$ means that in terms of RDM arithmetic we have $x^{\text {poss }}=\underline{x}+\alpha_{x}(\bar{x}-\underline{x})=1+2 \alpha_{x}$ and $y^{\text {poss }}=\underline{y}+\alpha_{y}(\bar{y}-\underline{y})=2+3 \alpha_{y}$. From the above equation, the following condition can be derived:

$$
\begin{aligned}
& \alpha_{y}=\left[\underline{x}-\underline{y}+\alpha_{x}(\bar{y}-\underline{y})\right] /(\bar{y}-\underline{y}), \\
& \alpha_{y}=-1 / 3+2 / 3 \cdot \alpha_{x} \\
& \alpha_{x}, \alpha_{y} \in[0,1], \quad \operatorname{card} X^{\text {poss }}, \quad \operatorname{card} Y^{\text {poss }}=c .
\end{aligned}
$$

Thus, when the relation $x=y$ holds, a mathematical model of addition is given by

$$
\begin{aligned}
& Z^{\mathrm{pr}}: z^{\mathrm{pr}}=x^{\mathrm{pr}}\left(\alpha_{x}\right)+y^{\mathrm{pr}}\left(\alpha_{y}\right), \\
& \alpha_{x}, \alpha_{y} \in[0,1], \quad \operatorname{card} X^{\mathrm{pr}}, \quad \operatorname{card} Y^{\mathrm{pr}}=1, \\
& \alpha_{y}=\left[\underline{x}-\underline{y}+\alpha_{x}(\bar{y}-\underline{y})\right] /(\bar{y}-\underline{y}), \\
& Z^{\mathrm{poss}}: z^{\mathrm{pos}}=x^{\mathrm{poss}}\left(\alpha_{x}\right)^{\mathrm{poss}}\left(\alpha_{y}\right), \\
& \alpha_{x}, \alpha_{y} \in[0,1], \quad \operatorname{card} X^{\mathrm{poss}}, \quad \operatorname{card} Y^{\mathrm{poss}}=c, \\
& \alpha_{y}=\left[\underline{x}-\underline{y}+\alpha_{x}(\bar{y}-\underline{y})\right] /(\bar{y}-\underline{y}) .
\end{aligned}
$$

Figure 7 shows a visualization of the set $Z^{\text {poss }}$ of fully dependent addition.

It should be noticed that in the case of the given example of addition of interval-valued variables $x$ and $y$, 


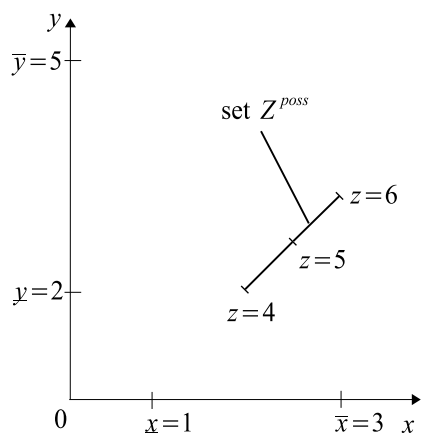

Fig. 7. Set $Z^{\text {poss }}$ of possible point results of addition of fully dependent variable values $x^{\text {poss }} \in[1,3]$ and $y^{\text {poss }} \in[2,5]$ under condition $y=x$ in projection on the space $X \times Y$.

with an increase in dependence sharpness (independency of $x$ and $y, y \geq x, y=x$ ), the cardinality of the set $Z^{\text {poss }}$ decreases (see Figs. 3, 6 and 7). The geometric irregularity of the set $Z^{\text {poss }}$ strongly hinders determining representatives of this set. In the case of more complicated mathematical dependencies, analytical determination of representatives of the set $Z^{\text {poss }}$ becomes impractical and numerical, approximate computer simulation methods should be applied. Multidimensional RDM I arithmetic presented in this section should be used according to the steps given below:

1. On the basis of the given, original form of mathematical equation/equations, derive a formula for the variable/parameter of interest by appropriate transformations of the original equation/equations.

2. Construct RDM models of all uncertain, interval-valued variables/parameters containing RDM variables $\alpha$.

3. Construct a mathematical model of the precise (true) value of the variable/parameter of interest. Substitute RDM models of uncertain variables/parameters from Step 2 into the formula obtained in Step 1.

4. Check whether the equation/equations elaborated in Step 3 satisfies the universality condition.

5. Construct a mathematical model of the set of possible point solutions. Transform the model of the precise value of the variable/variables of interest into the model of uncertainty of this value, the model of possible point solutions.

6. On the basis of the model from Step 5 using the analytical approach, determine precise representatives, or using the computer simulation approach determine approximate representatives (indicators) of the set of possible point solutions such as, e.g., the span, the cardinality measure distribution, the center of gravity of the set, etc.

\section{Examples of application of RDM interval arithmetic}

Example 1. Let us consider equation $[a]+X=[b]$. A real system is ruled by dependence $a+x=b$, where $a$ and $x$ are the inputs and $b$ is the output of the system. We know only an approximate value of $a \in[1,3]$ and $b \in[4,5]$, and we want to determine the value of the second input $x$. In terms of SI arithmetic, the following equation is to be solved:

$$
\begin{array}{r}
{[a]+[x]=[b], \quad[\underline{a}, \bar{a}]+[\underline{x}, \bar{x}]=[\underline{b}, \bar{b}],} \\
{[1,3]+X=[4,5] .}
\end{array}
$$

Solving such equations is not as trivial as it could seem, and many papers have been devoted to this subject both in terms of interval and fuzzy arithmetic (e.g., Mazarhuiya et al., 2011; Kovalerchuk and Kreinovich, 2016). If we assume that the solution of the equation is an interval, then with the use of SIA, on the basis of (48), we have $\underline{a}+\underline{x}=\underline{b}, \bar{a}+\bar{x}=\bar{b}$, which gives the solution $[\underline{x}, \bar{x}]=[3,2]$ being an improper interval. Such a solution, according to Lodwick and Dubois (2015), should be rejected as unrealistic. With the use of IIA and CIA, which possess the inverse element of addition, the term $[\underline{a}, \bar{a}]=[1,3]$ on the left-hand side of Eqn. (48) can be reduced, which gives solution $[\underline{x}, \bar{x}]=[\underline{b}, \bar{b}]-[\underline{a}, \bar{a}]=$ $[1,4]$. Is this solution the universal algebraic solution of (48) that satisfies all possible extension forms of the system equation (48)? There exist four different forms of the equation $a+x=b: a+x=b, a=b-x, x=$ $b-a, a+x-b=0$. It is easy to check that the solution $[\underline{x}, \bar{x}]=[1,4]$ is not the universal algebraic solution (UAS/UA solution) because it gives us the equality of the left- and right-hand sides only in the case of the extension $[a]+[x]=[b]$ and not for other extensions. For example, in the case of the extension $[a]=[b]-[x]$, we have $[1,3]=[4,5]-[1,4]$. According to IIA and CIA the $\mathrm{RH}$ side is equal to $[4,5]-[1,4]=[0,4]$. Because the $\mathrm{LH}$ side of the equation is equal to $[1,3]$, the $\mathrm{RH}$ side is different from the LH side. For comparison, a solution of the equation with RDM I arithmetic is given below.

Step 1. The crisp dependence ruling the system and deriving the formula for the variable of interest,

$$
a+x=b \rightarrow x=b-a .
$$

Step 2. RDM models of interval-valued variables,

$$
\begin{aligned}
& a \in[1,3]: a^{\mathrm{pr}}=1+2 \alpha_{a}, \\
& \alpha_{a} \in[0,1], \quad \operatorname{card} A^{\mathrm{pr}}=1 \\
& b \in[4,5]: b^{\mathrm{pr}}=4+\alpha_{b}, \\
& \quad \alpha_{b} \in[0,1], \quad \operatorname{card} B^{\mathrm{pr}}=1 .
\end{aligned}
$$


Step 3. The RDM model of the precise value of the variable of interest $x$,

$$
\begin{aligned}
& x^{\mathrm{pr}}=b^{\mathrm{pr}}-a^{\mathrm{pr}}=\left(4+\alpha_{b}\right)-\left(1+2 \alpha_{a}\right), \\
& x^{\mathrm{pr}}=3-2 \alpha_{a}+\alpha_{b}, \\
& \quad \alpha_{a}, \alpha_{b} \in[0,1], \quad \operatorname{card} A^{\mathrm{pr}}, \quad \operatorname{card} B^{\mathrm{pr}}=1 .
\end{aligned}
$$

Step 4. Verification of the universality of the RDM solution $x^{\mathrm{pr}}$ for all possible extension forms of the system equation $[a]+x=[b], x=[b]-[a],[a]=[b]-x$, $[a]+x-[b]=0$. Simple calculations prove that the RDM model of the precise solution $x^{\mathrm{pr}}\left(\alpha_{a}, \alpha_{b}\right)$ gives us the equality of the LH and RH sides of all extensions of the system equation. For example, for the form $[a]=[b]-x$ we have

$\left(1+2 \alpha_{a}\right)=\left(4+\alpha_{b}\right)-\left[\left(4+\alpha_{b}\right)-\left(1+2 \alpha_{a}\right)\right]=1+2 \alpha_{a}$.

This means that the $3 \mathrm{D}$ solution $x^{\mathrm{pr}}=3-2 \alpha_{a}+\alpha_{b}$ is the universal algebraic solution.

Step 5. The model of the set $X^{\text {poss }}$ of possible point-solutions,

$$
\begin{aligned}
& X^{\text {poss }}: x^{\text {poss }}=b^{\text {poss }}-a^{\text {poss }}=3-2 \alpha_{a}+\alpha_{b}, \\
& \alpha_{a}, \alpha_{b} \in[0,1], \quad \operatorname{card} A^{\text {poss }}, \quad \operatorname{card} B^{\text {poss }}=c .
\end{aligned}
$$

Figure 8 shows the set $X^{\text {poss }}$ in the 3D-space $A \times B \times$ $X$ and Fig. 9 its projection on the 2D-space $A \times B$.

Step 6. Determining representatives of the set $X^{\text {poss }}$.

The set $X^{\text {poss }}$ is generated by the formula $x^{\text {poss }}=$ $3-2 \alpha_{a}+\alpha_{b}$ with $\alpha_{a}, \alpha_{b} \in[0,1]$ and card $\alpha_{a}=c$, card $\alpha_{b}=c$ (see Step 5). If we are interested in the span $s\left(X^{\text {poss }}\right)$ being a measure (indicator) of its uncertainty, then we can easily determine it analytically as $\min x^{\text {poss }}=$ 1 for $\alpha_{a}=1$ and $\alpha_{b}=0$, and $\max x^{\text {poss }}=4$ for $\alpha_{a}=0$ and $\alpha_{b}=1$. Hence, the span is determined by the interval $s\left(X^{\text {poss }}\right)=[1,4]$. A representative of the set $X^{\text {poss }}$ in the form of a distribution of the cardinality measure $\operatorname{card} M(x)$ of particular subsets $x=$ const can be easily determined in this simple example on the basis of Fig. 9 by calculating lengths of particular isoclines corresponding to particular values $x=$ const (Fig. 10).

The distribution of card $M(x)$ is much more informative that span $s\left(X^{\text {poss }}\right)$ alone because it gives additional information about the frequency of particular possible result values $x$. This distribution can be given both in non-normalized and in normalized form with $\max \operatorname{card} M(x)=1$. We can also be interested in the representative of the set $X^{\text {poss }}$ in the form of position $x_{\text {CofG }}$ of its center of gravity CofG. This position can be calculated on the basis of the distribution of card $M$ with

$$
x_{\mathrm{CofG}}=\frac{\int_{\min x}^{\max x} x \cdot \operatorname{card} M(x) \mathrm{d} x}{\int_{\min x}^{\max x} \operatorname{card} M(x) \mathrm{d} x}=2.5 .
$$

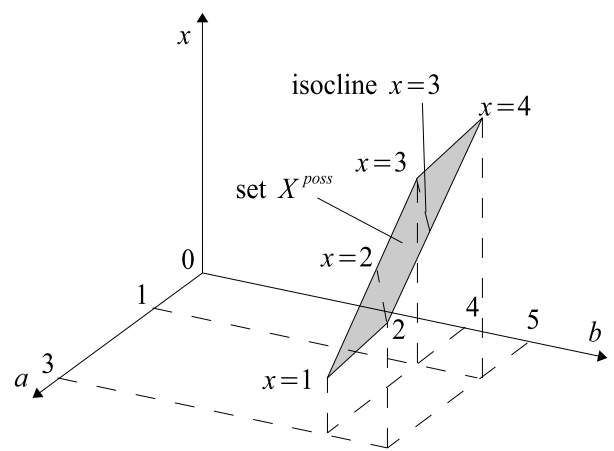

Fig. 8. Set $X^{\text {poss }}$ of possible point-solutions of equation $a^{\text {poss }}+$ $x^{\text {poss }}=b^{\text {poss }}$ for $a^{\text {poss }} \in[1,3], b^{\text {poss }} \in[4,5]$ in the $3 \mathrm{D}$ space $A \times B \times X$.

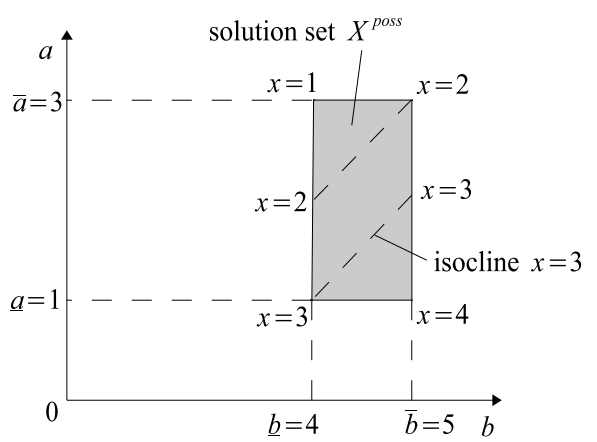

Fig. 9. Set $X^{\text {poss }}$ of possible point-solutions of equation $a^{\text {poss }}+$ $x^{\text {poss }}=b^{\text {poss }}$ for $a^{\text {poss }} \in[1,3], b^{\text {poss }} \in[4,5]$ in projection from the $3 \mathrm{D}$-space $A \times B \times X$ on the 2D-space $A \times B$.

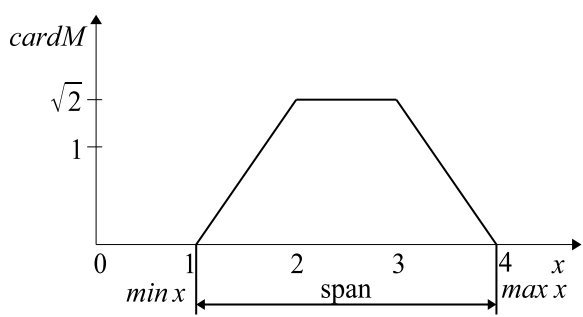

Fig. 10. Distribution of non-normalized cardinality measure card $M(x)$ of set $X^{\text {poss }}$ of possible point solutions. 


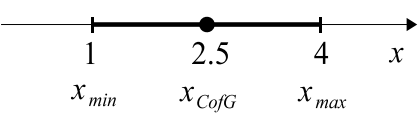

Fig. 11. Position $x_{\mathrm{CofG}}$ of the center of gravity and span $s\left(X^{\text {poss }}\right)$ of set $X^{\text {poss }}$ as representatives of set $X^{\text {poss }}$.

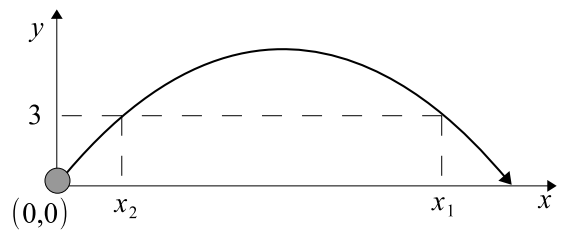

Fig. 12. Trajectory of the object after the start from $(x, y)=$ $(0,0)$ in the space $X \times Y$.

It is more informative to show the center of gravity against a background of the span of the set $X^{\text {poss }}$ than the center alone (Fig. 11).

Example 2. An object starts from point $(x, y)=(0,0)$ (Fig.12). Its acceleration is constant and known precisely, $g=9.8 \mathrm{~m} / \mathrm{s}^{2}$. Its initial velocity $u$ in the $x$ direction is known only approximately from measurements: $u \in U=$ $[10,15] \mathrm{m} / \mathrm{s}$. Similarly, its velocity $v$ in the $y$ direction $v \in V=[12,18] \mathrm{m} / \mathrm{s}$. Separate formulas determining the object position after time $t[\mathrm{~s}]$ are given by

$$
x=u t, \quad y=v t-0.5 g t^{2},
$$

and in aggregated form by

$$
\frac{-g x^{2}}{2 u^{2}}+\frac{v x}{u}-y=0 .
$$

Calculation task. Determine the distance $x$ from the initial point when the object will be on a height of $y=3 \mathrm{~m}$ (see Fig. 12).

Step 1. Determining the crisp dependence governing the system and deriving a formula for the variable of interest. For $y=3 \mathrm{~m}$, the formula (51) takes the form

$$
\frac{-g x^{2}}{2 u^{2}}+\frac{v x}{u}-3=0 .
$$

It is a quadratic equation that in general possesses two possible solutions, $x_{1}$ and $x_{2}$, containing $\Delta$ in the form

$$
\Delta=\frac{v^{2}-58.8}{u^{2}} .
$$

The possible solutions $x_{1}$ and $x_{2}$ for crisp data are determined as

$$
\begin{aligned}
& x_{1}=\frac{u}{g}\left[v+\left(v^{2}-58.8\right)\right]^{1 / 2}, \\
& x_{2}=\frac{u}{g}\left[v-\left(v^{2}-58.8\right)\right]^{1 / 2} .
\end{aligned}
$$

Step 2. Determining the RDM models of interval-valued variables. The interval-valued variables are velocities $u$ and $v: u \in[10,15]$ and $v \in[12,18] \mathrm{m} / \mathrm{s}$. The RDM models of the precise values of these velocities are determined by

$$
\begin{aligned}
& U^{\mathrm{pr}}: u^{\mathrm{pr}}=10+5 \alpha_{u}, \alpha_{u} \in[0,1], \\
& V^{\mathrm{pr}}: v^{\mathrm{pr}}=12+6 \alpha_{v}, \alpha_{v} \in[0,1], \\
& \operatorname{card} U^{\mathrm{pr}}=1, \quad \text { card } V^{\mathrm{pr}}=1 .
\end{aligned}
$$

Step 3. Determining RDM models of the precise values of the variables of interest $x_{1}$ and $x_{2}$ (roots of the quadratic equation (52),

$$
\begin{aligned}
X_{1}^{\mathrm{pr}}: x_{1}^{\mathrm{pr}}= & 0.10204\left(10+5 \alpha_{u}\right) \\
& \left\{\left(12+6 \alpha_{v}\right)+\left[\left(12+6 \alpha_{v}\right)^{2}-58.8\right]^{0.5}\right\}, \\
X_{2}^{\mathrm{pr}}: x_{2}^{\mathrm{pr}}= & 0.10204\left(10+5 \alpha_{u}\right) \\
\left\{\left(12+6 \alpha_{v}\right)-\left[\left(12+6 \alpha_{v}\right)^{2}-58.8\right]^{0.5}\right\}, & \quad \text { card } X_{1}^{\mathrm{pr}}, \quad \text { card } X_{2}^{\mathrm{pr}}=1 .
\end{aligned}
$$

Models $x_{1}^{\mathrm{pr}}$ and $x_{2}^{\mathrm{pr}}$ should be used in possible further calculations.

Step 4. Determining sets $X_{1}^{\text {poss }}$ and $X_{2}^{\text {poss }}$ of possible point solutions,

$$
\begin{aligned}
& X_{1}^{\text {poss }}: x_{1}^{\text {poss }}= 0.10204\left(10+5 \alpha_{u}\right) \\
&\left\{\left(12+6 \alpha_{v}\right)+\left[\left(12+6 \alpha_{v}\right)^{2}-58.8\right]^{0.5}\right\}, \\
& X_{2}^{\text {poss }}: x_{2}^{\text {poss }}= 0.10204\left(10+5 \alpha_{u}\right) \\
&\left\{\left(12+6 \alpha_{v}\right)-\left[\left(12+6 \alpha_{v}\right)^{2}-58.8\right]^{0.5}\right\}, \\
& \alpha_{u}, \alpha_{v} \in[0,1], \quad \operatorname{card} X_{1}^{\text {poss }}, \quad \operatorname{card} X_{2}^{\text {poss }}=c .
\end{aligned}
$$

Mathematical models of sets $X_{1}^{\text {poss }}$ and $X_{2}^{\text {poss }}$ allow generating both particular point solutions $x_{1}^{\text {poss }}$ and $x_{2}^{\text {poss }}$ and their representatives such as spans, frequency (cardinality) distributions, centers of gravity of solution sets, and other set features. These models also allow generating any possible system state by assuming numeric values of RDM variables $\left(\alpha_{u}, \alpha_{v}\right)$. For example, variable values $\alpha_{u}=0.3, \alpha_{v}=0.5$ correspond to the system state: $u^{\text {poss }}=11.5, v^{\text {poss }}=15, x_{1}^{\text {poss }}=32.73000$, $x_{2}^{\text {poss }}=2.47381$. Such a single system state can be called a point-state because it creates a point in the system space $U \times V \times X_{1} \times X_{2}$, and the solutions $x_{1}^{\text {poss }}=$ $32.73000, x_{2}^{\text {poss }}=2.47381$ can appropriately be called point-solutions in the solution space $X_{1}^{\text {poss }} \times X_{2}^{\text {poss }}$.

Step 5. Determining representatives of possible solution sets $X_{1}^{\text {poss }}$ and $X_{2}^{\text {poss }}$.

The spans $s\left(X_{1}^{\text {poss }}\right)$ can be determined analytically by examining (57) because of the function simplicity; $\min \left(x_{1}^{\text {poss }}\right)=21.673$ occurs for $\alpha_{u}=\alpha_{v}=0$ and $\max \left(x_{1}^{\text {poss }}\right)=52.487$ occurs for $\alpha_{u}=\alpha_{v}=1$. Hence, the span $s\left(X_{1}^{\text {poss }}\right)=[21.673,52.487]$. Analytical determination of the span $s\left(X_{2}^{\text {poss }}\right)$ is more difficult, 
$y^{4}$

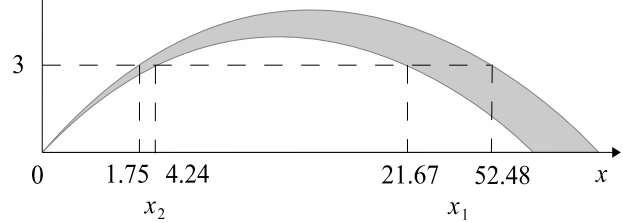

Fig. 13. Visualisation of uncertainty of the object trace and of the sense of calculated spans $s\left(X_{1}^{\text {poss }}\right)=$ $[21.67,52.49]$ and $s\left(X_{2}^{\text {poss }}\right)=[1.75,4.24]$.

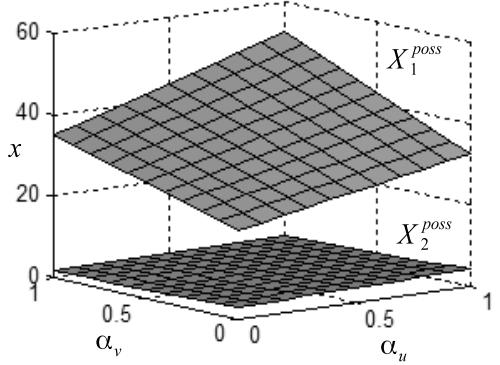

Fig. 14. Visualization of sets $X_{1}^{\text {poss }}$ and $X_{2}^{\text {poss }}$ of possible pointsolutions $x_{1}^{\text {poss }}$ and $x_{2}^{\text {poss }}$ of the object position $x$ at the height $y=3 \mathrm{~m}$.

therefore its function given by (57) has been examined by simulation; $\min \left(x_{2}^{\text {poss }}\right)=1.75$ occurs for $\alpha_{u}=0$ and $\alpha_{v}=1$ and $\max \left(x_{2}^{\text {poss }}\right)=4.24$ occurs for $\alpha_{u}=1$ and $\alpha_{v}=0$. Hence, the span $s\left(X_{2}^{\text {poss }}\right)=[1.75,4.24]$. Figure 13 illustrates uncertainty of the possible object trace.

The spans $s\left(X_{1}^{\text {poss }}\right), s\left(X_{2}^{\text {poss }}\right)$ themselves are only general and insufficient information about possible roots $x_{1}^{\text {poss }}$ and $x_{2}^{\text {poss }}$ of the system equation (52). Note that the roots of the quadratic equation are complex conjugate and the correct result has to inform about pairs of complex conjugate roots $\left(x_{1}^{\text {poss }}, x_{2}^{\text {poss }}\right)$. This coupling is only possible by RDM variables $\alpha_{u}, \alpha_{v}$, as shown in the formulas (57).

The given problem of solution sets $X_{1}^{\text {poss }}$ and $X_{2}^{\text {poss }}$ is three-dimensional and can be visualized. Figure 14 shows two sets of possible point-solutions $x_{1}^{\text {poss }}$ and $x_{2}^{\text {poss }}$. Here $\alpha_{u}$ and $\alpha_{v}$ are independent RDM variables that are transformable in variables $u$ and $v$ according to (55).

The next representative of the possible solution sets $X_{1}^{\text {poss }}$ and $X_{2}^{\text {poss }}$ is the frequency (un-normalized cardinality) distribution of possible root values $x_{1}^{\text {poss }}$ and $x_{2}^{\text {poss }}$. To determine it analytically, intricate integrals have to be calculated. A more practical way is computer simulation with the use of Matlab in which $10^{7}$ of random values of RDM variables $\alpha_{u}, \alpha_{v}$ from interval $[0,1]$ have been generated and the corresponding root pairs $\left(x_{1}^{\text {poss }}, x_{2}^{\text {poss }}\right)$ were calculated according to (57). Figure 15



Fig. 15. Distribution of $10^{7}$ of random solutions (roots, values) $x_{1}^{\text {poss }}$ and $x_{2}^{\text {poss }}$ of Eqn. 51) describing the position $x$ of the object at the height $y=3 \mathrm{~m}$.

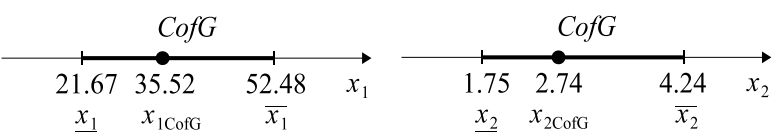

Fig. 16. Positions $x_{1 \mathrm{CofG}}$ and $x_{2 \mathrm{CofG}}$ of the centers of gravity of possible solution sets $X_{1}^{\text {poss }}$ and $X_{2}^{\text {poss }}$ against a background of their spans $s\left(X_{1}^{\text {poss }}\right)$ and $s\left(X_{2}^{\text {poss }}\right)$.

shows the number of occurrences of a particular root's values (histogram).

The next simplified representative of the possible solution sets $X_{1}^{\text {poss }}$ and $X_{2}^{\text {poss }}$ can be positions $x_{1 \text { CofG }}$ and $x_{2 \text { CofG }}$ of centers of gravity (expected values of particular roots $x_{1}^{\text {poss }}$ and $\left.x_{2}^{\text {poss }}\right)$. According to the calculations made on the basis of the simulation results $x_{1 \mathrm{CofG}}=2.74$ and $x_{2 \mathrm{CofG}}=35.52$. Figure 16 shows the centers of gravity of the solutions against the background of their spans.

Example 3. The task now consists in determining solutions $x_{1}$ and $x_{2}$ of the interval linear-equation system (58),

$$
\begin{aligned}
& {\left[a_{1}\right] x_{1}+\left[a_{2}\right] x_{2}=\left[a_{3}\right],} \\
& {\left[a_{4}\right] x_{1}+\left[a_{5}\right] x_{2}=\left[a_{6}\right],} \\
& {[2,4] x_{1}+[-2,1] x_{2}=[-2,2],} \\
& {[-1,2] x_{1}+[2,4] x_{2}=[-2,2] .}
\end{aligned}
$$

This system of equations can have the meaning of a balance model of a mechanical, biological or economic, system. In the case of economic systems, linear balance models were introduced by Dymova (2011), Leontief (1966) or Sevastjanov and Dymova (2009), and are frequently called Leontief's models. In (58), we can notice pairs of equal intervals, e.g., $\left[a_{1}\right]=[2,4]$ and $\left[a_{5}\right]=[2,4]$. However, the mathematical equality of intervals $[2,4]=[2,4]$ does not mean that the true values of uncertain parameters $\left[a_{1}\right]$ and that $\left[a_{5}\right]$ are equal. In a real system, the true value of $a_{1}$ can be 2.11 and that of 
$a_{5}$ can be 3.97. Therefore, these parameters have to be modeled as different variables.

Step 1. Determining the crisp dependence governing the system on the basis of the well-known Cramer formulas and deriving the formulas (59) for the variables of interest $x_{1}$ and $x_{2}$,

$$
x_{1}=\frac{a_{3} a_{5}-a_{6} a_{6}}{a_{1} a_{5}-a_{2} a_{4}}, \quad x_{2}=\frac{a_{1} a_{2}-a_{3} a_{4}}{a_{1} a_{5}-a_{2} a_{4}} .
$$

Step 2. Determining the RDM models (60) of interval-valued parameters,

$$
\begin{gathered}
{\left[a_{1}\right]=[2,4]: A_{1}^{\mathrm{pr}}: a_{1}^{\mathrm{pr}}=2+2 \alpha_{1},} \\
\alpha_{1} \in[0,1], \quad \operatorname{card} A_{1}^{\mathrm{pr}}=1, \\
{\left[a_{2}\right]=[-2,1]: A_{2}^{\mathrm{pr}}: a_{2}^{\mathrm{pr}}=-2+3 \alpha_{2},} \\
\alpha_{2} \in[0,1], \quad \operatorname{card} A_{2}^{\mathrm{pr}}=1, \\
{\left[a_{3}\right]=[-2,2]: A_{3}^{\mathrm{pr}}: a_{3}^{\mathrm{pr}}=-2+4 \alpha_{3},} \\
\alpha_{3} \in[0,1], \quad \operatorname{card} A_{3}^{\mathrm{pr}}=1, \\
{\left[a_{4}\right]=[-1,2]: A_{4}^{\mathrm{pr}}: a_{4}^{\mathrm{pr}}=-1+3 \alpha_{4},} \\
\alpha_{4} \in[0,1], \quad \operatorname{card} A_{4}^{\mathrm{pr}}=1, \\
{\left[a_{5}\right]=[2,4]: A_{5}^{\mathrm{pr}}: a_{5}^{\mathrm{pr}}=2+2 \alpha_{5},} \\
\alpha_{5} \in[0,1], \quad \operatorname{card} A_{5}^{\mathrm{pr}}=1, \\
{\left[a_{6}\right]=[-2,2]: A_{6}^{\mathrm{pr}}: a_{6}^{\mathrm{pr}}=-2+4 \alpha_{6},} \\
\alpha_{6} \in[0,1], \quad \operatorname{card} A_{6}^{\mathrm{pr}}=1 .
\end{gathered}
$$

Step 3. Determining the RDM models 61 of the precise values of variables of interest $x_{1}$ and $x_{2}$,

$$
\begin{aligned}
& X_{1}^{\mathrm{pr}}: x_{1}^{\mathrm{pr}} \\
& =\frac{\left(-2+4 \alpha_{3}\right)\left(2+2 \alpha_{5}\right)-\left(-2+3 \alpha_{2}\right)\left(-2+4 \alpha_{6}\right)}{\left(2+2 \alpha_{1}\right)\left(2+2 \alpha_{5}\right)-\left(-2+3 \alpha_{2}\right)\left(-1+3 \alpha_{4}\right)}, \\
& X_{2}^{\mathrm{pr}}: x_{2}^{\mathrm{pr}} \\
& =\frac{\left(2+2 \alpha_{1}\right)\left(-2+4 \alpha_{6}\right)-\left(-2+4 \alpha_{3}\right)\left(-1+3 \alpha_{4}\right)}{\left(2+2 \alpha_{1}\right)\left(2+2 \alpha_{5}\right)-\left(-2+3 \alpha_{2}\right)\left(-1+3 \alpha_{4}\right)}, \\
& \alpha_{1}, \ldots, \alpha_{6} \in[0,1], \quad \operatorname{card} X_{1}^{\mathrm{pr}}=1, \quad \operatorname{card} X_{2}^{\mathrm{pr}}=1 .
\end{aligned}
$$

Step 4. Checking the correctness of solutions (61). Substituting the solutions (61) in the system (58), we check that they satisfy it .

Step 5. Determining the RDM models (62) of sets $X_{1}^{\text {poss }}$ and $X_{2}^{\text {poss }}$ of possible point-solutions,

$$
\begin{aligned}
& X_{1}^{\text {poss }}: x_{1}^{\text {poss }} \\
& =\frac{\left(-2+4 \alpha_{3}\right)\left(2+2 \alpha_{5}\right)-\left(-2+3 \alpha_{2}\right)\left(-2+4 \alpha_{6}\right)}{\left(2+2 \alpha_{1}\right)\left(2+2 \alpha_{5}\right)-\left(-2+3 \alpha_{2}\right)\left(-1+3 \alpha_{4}\right)}, \\
& X_{2}^{\text {poss }}: x_{2}^{\text {poss }} \\
& =\frac{\left(2+2 \alpha_{1}\right)\left(-2+4 \alpha_{6}\right)-\left(-2+4 \alpha_{3}\right)\left(-1+3 \alpha_{4}\right)}{\left(2+2 \alpha_{1}\right)\left(2+2 \alpha_{5}\right)-\left(-2+3 \alpha_{2}\right)\left(-1+3 \alpha_{4}\right)}, \\
& \alpha_{1}, \ldots, \alpha_{6} \in[0,1], \quad \text { card } X_{1}^{\text {poss }}=c, \quad \text { card } X_{2}^{\text {poss }}=c .
\end{aligned}
$$

Examining the determinant of the system $(58)$ being the denominator in these equations for various values of RDM variables $\alpha_{1}, \ldots, \alpha_{6}$, shows that the determinant is always positive

$$
\begin{aligned}
2 \leq & \left(2+2 \alpha_{1}\right)\left(2+2 \alpha_{5}\right) \\
& -\left(-2+3 \alpha_{2}\right)\left(-1+3 \alpha_{4}\right) \leq 20 .
\end{aligned}
$$

This means that both sets $X_{1}^{\text {poss }}$ and $X_{2}^{\text {poss }}$ of possible point-solutions are 1-granular and not multi-granular as in the case of changing determinant from - to + (Piegat and Plucinski, 2017). Equations (62) allow generating each possible, complex conjugate point-solution pair $\left(x_{1}, x_{2}\right)$ of the system (58). For example, for $\alpha_{1}=\alpha_{3}=\alpha_{5}=$ $\alpha_{6}=0.5$ and $\alpha_{2}=\alpha_{4}=0$, coefficients $a_{i}$ take on the following values: $a_{1}=3, a_{2}=-2, a_{3}=0, a_{4}=-1$, $a_{5}=3, a_{6}=0$. For these parameter values, Eqns. (58) take the form of

$$
3 x_{1}-2 x_{2}=0, \quad-x_{1}+3 x_{2}=0 \text {. }
$$

The solutions calculated with the formulas systems of (62) have the values $x_{1}=x_{2}=0$ and satisfy (58) and (64). For other values of RDM variables $\alpha_{1}, \ldots, \alpha_{6}$, other values of system parameters $a_{1}, \ldots, a_{6}$ and other solution pairs $\left(x_{1}, x_{2}\right)$ are achieved. Solutions $x_{1}$ and $x_{2}$ are coupled together by variables $\alpha_{1}, \ldots, \alpha_{6}$. Thus, they are dependent. This dependence can be expressed by

$$
\begin{aligned}
x_{2}= & x_{1} \\
& \times \frac{\left(2+2 \alpha_{1}\right)\left(-2+4 \alpha_{6}\right)-\left(-2+4 \alpha_{3}\right)\left(-1+3 \alpha_{4}\right)}{\left(-2+4 \alpha_{3}\right)\left(2+2 \alpha_{5}\right)-\left(-2+3 \alpha_{2}\right)\left(-2+4 \alpha_{6}\right)} .
\end{aligned}
$$

For various sextuples $\left(\alpha_{1}, \ldots, \alpha_{6}\right)$, different solution pairs $\left(x_{1}, x_{2}\right)$ are achieved. The set of these pairs is displayed in Fig. 17

Step 6. Determining representatives of sets $X_{1}^{\text {poss }}$ and $X_{2}^{\text {poss }}$ of possible point-solutions $x_{1}^{\text {poss }}$ and $x_{2}^{\text {poss }}$. If we are interested in spans $s\left(X_{1}^{\text {poss }}\right)$ and $s\left(X_{2}^{\text {poss }}\right)$ of sets of possible point-solutions, then they can be determined with an analytical method (function examining) or with a computer simulation method on the basis of

$$
\begin{aligned}
& s\left(X_{1}^{\text {poss }}\right)=\left[\min _{\alpha_{1}, \ldots, \alpha_{6}} x_{1}^{\text {poss }}, \max _{\alpha_{1}, \ldots, \alpha_{6}} x_{1}^{\text {poss }}\right]=[-4,4], \\
& s\left(X_{2}^{\text {poss }}\right)=\left[\min _{\alpha_{1}, \ldots, \alpha_{6}} x_{2}^{\text {poss }}, \max _{\alpha_{1}, \ldots, \alpha_{6}} x_{2}^{\text {poss }}\right]=[-4,4],
\end{aligned}
$$

where $x_{1}^{\text {poss }}$ and $x_{2}^{\text {poss }}$ are determined by the formulas (62).

The value of $\min x_{1}^{\text {poss }}$ was achieved for $\alpha_{1}=0$, $\alpha_{2}=0, \alpha_{3}=0, \alpha_{4}=0, \alpha_{5}=0, \alpha_{6}=0$, the value of $\max x_{1}^{\text {poss }}$ for $\alpha_{1}=0, \alpha_{2}=0, \alpha_{3}=1, \alpha_{4}=0, \alpha_{5}=0$, $\alpha_{6}=1$, the value of $\min x_{2}^{\text {poss }}$ for $\alpha_{1}=0, \alpha_{2}=1$, $\alpha_{3}=1, \alpha_{4}=1, \alpha_{5}=0, \alpha_{6}=0$, and the value of $\max$ 


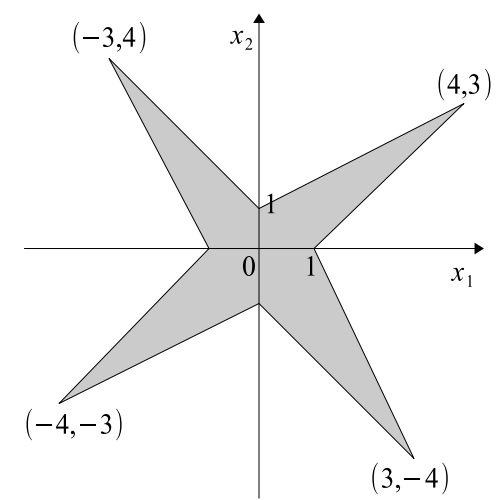

Fig. 17. Visualization of pairs $\left(x_{1}, x_{2}\right)$ of possible solutions of the system (58) achieved by computer generated solutions for various sextuples of RDM variables $\left(\alpha_{1}, \ldots, \alpha_{6}\right)$.

\begin{tabular}{|c|c|c|c|c|c|c|c|}
\hline \multicolumn{4}{|c|}{$\operatorname{Cof} G$} & \multicolumn{4}{|c|}{$\operatorname{Cof} G$} \\
\hline-4 & 0 & 4 & $x_{1}$ & -4 & 0 & 4 & $x_{2}$ \\
\hline$\underline{x_{1}}$ & $x_{1 \mathrm{CofG}}$ & $\overline{x_{1}}$ & & $\underline{x_{2}}$ & $x_{2 \mathrm{CofG}}$ & $\overline{x_{2}}$ & \\
\hline
\end{tabular}

Fig. 18. Representatives of possible solutions sets $X_{1}^{\text {poss }}$ and $X_{2}^{\text {poss }}$ in the form of their centers of gravity (expected values) $x_{1 \text { CofG }}$ and $x_{2 \text { CofG }}$ vs. a background of their spans $s\left(X_{1}^{\text {poss }}\right)$ and $s\left(X_{2}^{\text {poss }}\right)$.

$x_{2}^{\text {poss }}$ for $\alpha_{1}=0, \alpha_{2}=1, \alpha_{3}=0, \alpha_{4}=1, \alpha_{5}=0$, $\alpha_{6}=1$. The achieved spans $s\left(X_{1}^{\text {poss }}\right)=[-4,4]$ and $s\left(X_{2}^{\text {poss }}\right)=[-4,4]$ of the possible solution sets should convince everyone that spans are not solutions of a system of equations because only appropriately chosen pairs of point-solutions $x_{1}^{\text {poss }}$ and $x_{2}^{\text {poss }}$ satisfy this system. The values of point-solutions cannot be chosen freely from particular span-intervals. They should satisfy Eqns. (58). If one is interested in positions $x_{1 \mathrm{CofG}}$ and $x_{2 \mathrm{CofG}}$ of the centers of gravity (expected values of $x_{1}$ and $x_{2}$ ) as representatives of possible solution sets $X_{1}^{\text {poss }}$ and $X_{2}^{\text {poss }}$, then, due to a high problem dimensionality, one should apply a computer simulation method which gives the result $x_{1 \text { CofG }}=0$ and $x_{2 \text { CofG }}=0$. Figure 18 shows the centers of gravity vs. the background of spans.

\section{Conclusions}

The paper shows that the right result of arithmetic operations on intervals is not an interval, i.e., not a one-dimensional information granule, but a multi-dimensional one. An interval can only be a representative of this granule delivering a simplified information about it. Two multidimensional result-granules can be different, but they can have the same representative in the form of, e.g., their span (interval). Hence, they will be indistinguishable in a 1D-space whereas they are distinguishable in their full, multi-dimensional space. Using the interval as a result of arithmetic operations on intervals frequently leads to unacceptable results observed in these calculations and described in the subject literature. The paper presents the correct way of realization of arithmetic operations and equation solving which prevents achieving unacceptable results in interval computations.

\section{References}

Bader, F. and Nipkow, T. (1998). Term Rewriting and All That, Cambridge University Press, Cambridge.

Birkhoff, G. (1967). Lattice Theory, Vol. XXV, Colloquium Publications, American Mathematical Society, Providence, RI.

Chalco-Cano, Y., Lodwick, W. and Bede, B. (2014). Single level constraint interval arithmetic, Fuzzy Sets and Systems 257: $146-168$.

Dabala, K. (2009). Research of possibilities of interval arithmetic application to induction motors efficiency determination, Zeszyty Problemowe: Maszyny Elektryczne (84): 39-44.

Dymova, L. (2011). Soft Computing in Economics and Finance, Springer, Berlin/Heidelberg.

Figuiredo, L. and Stolfi, J. (2004). Affine arithmetic: Concepts and applications, Numerical Algorithms 37(1): 147-158.

Hanss, M. (2005). Applied Fuzzy Arithmetic, Springer, Berlin/Heidelberg.

Hayes, B. (2003). A lucid interval, American Scientist 91(6): 484-488.

Kaucher, E. (1980). Interval analysis in the extended interval space IR, Computing Supplement 2: 33-49.

Kovalerchuk, B. and Kreinovich, V. (2016). Comparisons of applied tasks with intervals, fuzzy sets and probability approaches, Proceedings of the 2016 IEEE International Conference on Fuzzy Systems (FUZZ), Vancouver, Canada, pp. 1478-1483.

Leontief, W. (1949). The Structure of the American Economy, 1919-1935, Oxford University Press, London.

Leontief, W. (1966). Input-Output Economics, Oxford University Press, New York, NY.

Lodwick, W. (1999). Constrained interval arithmetic, Technical Report CCM, University of Colorado at Denver, Denver, $\mathrm{CO}$.

Lodwick, W. and Dubois, D. (2015). Interval linear systems as a necessary step in fuzzy linear systems, Fuzzy Sets and Systems 281: 227-251.

Lyashko, M. (2005). The optimal solution of an interval systems of linear algebraic equations, Reliable Computing 11(2): 227-251.

Mazarhuiya, F., Mahanta, A. and Baruah, H. (2011). Solution of fuzzy equation $a+x=b$ using method of superimposition, Applied Mathematics 2(8): 1039-1045. 
Moore, R. (1996). Interval Analysis, Prentice Hall, Englewood Cliffs, NJ.

Moore, R., Baker, K. and Cloud, M. (2009). Introduction to Interval Analysis, SIAM, Philadelphia, PA.

Moore, R. and Young, C. (1959). Interval analysis I, Technical Report LMSD285875, Lockheed Missiles and Space Division, Sunnyvale, CA.

Neumaier, A. (1990). Interval Methods for Systems of Equations, Cambridge University Press, Cambridge.

Pedrycz, W., Skowron, A. and Kreinovich, V. (2008). Handbook of Granular Computing, John Wiley\&Sons, Chichester.

Piegat, A. and Landowski, M. (2012). Is the conventional interval arithmetic correct?, Journal of Theoretical and Applied Computer Science 6(2): 27-44.

Piegat, A. and Landowski, M. (2013). Two interpretations of multidimensional RDM interval arithmetic-multiplication and division, International Journal of Fuzzy Systems 15(4): 488-496.

Piegat, A. and Pluciński, M. (2015). Computing with words with the use of inverse RDM models of membership functions, International Journal of Applied Mathematics and Computer Science 25(3): 675-688, DOI: 10.1515/amcs-2015-0049.

Piegat, A. and Plucinski, M. (2017). Fuzzy number division and the multi-granularity phenomenon, Bulletin of the Polish Academy of Sciences: Technical Sciences 65(4): 497-511.

Piegat, A. and Tomaszewska, K. (2013). Decision making under uncertainty using info-gap theory and a new multidimensional RDM interval arithmetic, Przeglad Elektrotechniczny 89(8): 71-76.

Pilarek, M. (2010). Solving systems of linear equations using the interval extended zero method and multimedia extensions, Scientific Research of the Institute of Mathematics and Computer Science 9(2): 203-212.

Popova, E. (1998). Algebraic solutions to a class of interval equations, Journal of Universal Computer Science 4(1): 48-67.

Sevastjanov, P. and Dymova, L. (2009). A new method for solving internal and fuzzy equations: Linear case, Information Sciences 179: 925-937.
Shary, S. (1996). Algebraic approach to the interval linear static identification, tolerance and control problems, or one more application of Kaucher arithmetic, Reliable Computing 2(1): 3-33.

Shary, S. (2002). A new technique in systems analysis under interval uncertainty and ambiguity, Reliable Computing 8: $321-418$.

Sunaga, T. (1958). Theory of an interval algebra and its application to numerical analysis, RAAG Memoirs 2: $547-564$.

Warmus, M. (1956). Calculus of approximations, Bulletin de l'Académie Polonaise des Sciences Cl. III 4(5): 253-259.

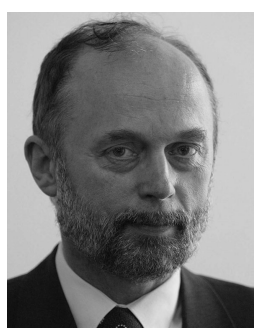

Andrzej Piegat received his $\mathrm{PhD}$ degree in modeling and control of production systems from the Technical University of Szczecin in 1979, the DSc degree in control of underwater vehicles from Rostock University in 1998, and the professorial title in 2001. At present he is a professor at the West Pomeranian University of Technology, Szczecin, Poland. His current research is focused on uncertainty theory, fuzzy logic, computing with words and info-gap theory.

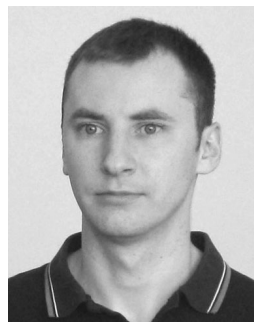

Marek Landowski received his MSc degrees in mathematics and in computer science in 2002 and 2004, respectively. In 2009 he obtained a PhD degree in identification of probabilistic models of human perception from the West Pomeranian University of Technology. Currently he is an assistant professor at the Maritime University of Szczecin, Poland. At present his research interests are focused on fuzzy arithmetic, data mining and uncertainty theory.

Received: 10 January 2017 Revised: 14 April 2017 Accepted: 27 May 2017 